\title{
How Clean is Clean Enough? Maintaining Thermal Protective Clothing Under Field Conditions in the Oil and Gas Sector
}

\author{
Elizabeth M. Crown \\ University of Alberta, Edmonton, Alta., Canada \\ Aifen Feng \\ Hebei University of Science and Technology, Shijiazhuang, P.R. China
}

\author{
Xia Xu \\ University of Alberta, Edmonton, Alta., Canada
}

The purpose of this research was to develop practical care procedures to help maintain the protective quality of flame resistant workwear laundered by workers in the field. Based on observed field conditions, experiments were conducted that simulated domestic laundry procedures. The first experiment involved two flame resistant (FR) fabrics, contaminated or not contaminated with oil. Independent variables also included detergent type and laundry pre-treatment. Other laundry parameters were controlled. Results indicated that it is easier to maintain the FR performance of the FR-treated blend than it is for the aramid fabric. It is hypothesized that energy generated by initial ignition of oil on the specimens triggers the FR mechanism of the treatment, which in turn inhibits further combustion. A second experiment using larger specimens and a domestic washing machine also supported the hypothesized mechanism.

$$
\text { protective clothing decontamination flammability }
$$

\section{INTRODUCTION}

The impetus for this research occurred when a worker wearing flame resistant (FR) coveralls was exposed to a flash fire and suffered third-degree burns to much of his body. Was the FR protective clothing inadequate, or had it not been properly cleaned to ensure adequate protection? FR clothing can protect a worker in flash fire situations if the clothing is kept clean and is free of combustible materials. Advice on appropriate maintenance is provided by most FR clothing producers, but such advice is not always practical in the field. Also, the degree of cleanliness required to maintain the protective qualities of FR clothing is not well known. Most laundry research on FR clothing has addressed the issue of durability of FR finishes. Stull [1] studied the effectiveness of several cleaning methods for structural firefighting protective clothing and found that dry cleaning and even aeration were most effective in removing common contaminants in such garments. Mäkinen [2] included measures of flammability and radiant heat transmission when studying the effect of wear and laundering on structural firefighters' worn garments. Worn dirty garments had greater

The researchers wish to acknowledge financial support for this project from Amoco Canada Petroleum Company (BP Amoco Gas Business Unit), AEC Oil and Gas, and the Department of Human Ecology, University of Alberta. We are also grateful to Dr Kathy Rigakis, Elaine Bitner and Kathryn Chandler for assistance with the field studies and laboratory experiments.

Correspondence and requests for offprints should be sent to Elizabeth M. Crown, 302 Human Ecology Building, Universtiy of Alberta, Edmonton, AB, T6G 2N1, Canada. E-mail: < betty.crown@ualberta.ca>. 
flammability than new garments but heat transmission was not significantly affected.

How dirty must a garment be before it fails to protect the worker? How clean is clean enough? To help answer these questions, a multi-phase research project was conducted. The purpose was to develop appropriate, practical care procedures to help maintain the protective properties of FR workwear over its useful lifetime. To gain an understanding of the working conditions and laundering procedures typical for many oil and gas sector workers who wear FR clothing, and of the barriers to keeping FR workwear clean, field sites were visited and employee interviews and a survey were conducted. The results of this early phase [3] indicated that, given typical working conditions, it is not possible to keep some FR garments completely clean, but that many improvements in consistency and quality can be made to the current cleaning procedures, including locating laundry facilities at all plant/field sites with clearly posted instructions for cleaning.

The second phase of the project [4] comprised a 5-month wear trial designed to determine if garments worn in the field in normal work environments could be cleaned satisfactorily under controlled laboratory conditions using currently recommended procedures. New garments fabricated from both aramid and FR viscose/aramid blend fabrics were worn by 5 employees according to a planned schedule, and after each day of wearing were sent to our laboratory for inspection, laundering and mending. Garments were removed from the trial for flame resistance testing after 1, 5, 10 and 15 wear/wash cycles. The aramid garments rated the dirtiest before laundering were the most likely to fail the flame resistance test after laundering, while none of the FR blend garments failed the flame resistance test after laundering. However, it was not clear that the blend garments were getting cleaner. Further investigation of this phenomenon was needed.

\section{EXPERIMENTAL METHOD}

This paper focuses on the experimental phase of the research that included preliminary experiments to establish some of the control and independent variables, a small-scale experiment using simulated laundry procedures and a final experiment with larger specimens laundered in a regular washing machine.

\subsection{Small-Scale Contamination/Decontamination Experiment}

Three replications of an experiment were run, in which independent variables included fabric, contamination, laundry detergent and pre-laundry treatment. The two FR fabrics used for coveralls in the earlier phase of the research were tested, namely a $209-\mathrm{g} / \mathrm{m}^{2}$ aramid and a $260-\mathrm{g} / \mathrm{m}^{2}$ FR viscose/aramid blend. Half the fabrics were contaminated with motor oil and half were laundered without contamination. The two detergents were an industrial powdered detergent commonly used in the industry, and one common domestic liquid. Fabric specimens were either given no pre-treatment before laundry or one of three different pre-treatments, namely a $10 \%$ solution of an industrial degreaser and two domestic pre-wash sprays. Dependent variables were measures of FR performance.

\subsubsection{Specimen preparation}

For each replication, sufficient specimens, $100 \mathrm{~mm}$ wide by $220 \mathrm{~mm}$ long, were cut from each fabric to randomly assign five specimens to each experimental treatment. For each contaminated specimen 18 drops $(0.4 \mathrm{~g})$ of Amoco's low-ash oil were dropped from a burette onto predetermined spots spread over the fabric surface. Contaminated specimens were allowed to age for 3 days prior to laundering. After laundering, specimens were trimmed to size for FR testing. 


\subsubsection{Laundry procedures}

Prior to washing, specimens to be pre-treated were fully soaked by spraying evenly with $9 \mathrm{ml}$ of the pre-treatment solution. Each specimen was added individually to a Launder-Ometer canister with detergent solution $(1.96 \mathrm{~g} / 200 \mathrm{ml}$ water) at $60^{\circ} \mathrm{C}$ and 25 steel balls. After $10 \mathrm{~min}$, the wash solution was drained from the canisters. Two rinse cycles of 5 and $3 \mathrm{~min}$ respectively followed, each with $200 \mathrm{ml}$ of water at $30{ }^{\circ} \mathrm{C}$. Following the second rinse, specimens were removed from the canisters and hung to air dry.

\subsubsection{Measurement of flame resistance}

Specimens were trimmed and tested for flame resistance one day after laundering, following CAN-CGSB-4.2, No. 27.10-2000, Flame resistance - vertically oriented textile fabric or fabric assembly test [5], using the edge ignition procedure. This method determines the burning behavior of vertically oriented flame resistant fabrics by applying a defined ignition flame to the edge of the specimen for $12 \mathrm{~s}$, with the burner inclined at $30^{\circ}$ to the vertical. Oven-dried specimens are tested as this more severe condition better simulates the worst-case dry conditions of Canadian winters. The burner, specimen frame and burner ignition location are the same as specified in ISO 6940:1984 [6]. After-flame (seconds), after-glow (seconds) and notable observations were recorded for each specimen, and damaged length was measured after specimens cooled. Only damaged length and after-flame were included in the analysis reported here.

\subsubsection{Statistical analysis}

Means and standard deviations were calculated for each experimental treatment for each fabric, and analyses of variance were conducted to determine differences among both fabrics and treatments as well as interaction effects among the experimental variables.

\subsection{Large-Scale \\ Contamination/Decontamination Experiments}

Two replications were run of an experiment with the same two fabrics as in the small-scale experiment. Larger samples were washed in a domestic washing machine to more closely simulate potential field conditions, and only the industrial detergent was used. Experimental variables were contaminant (oil or no oil), and pre-treatment (none, industrial degreaser solution, or domestic pre-wash spray).

Samples measuring $500 \times 220 \mathrm{~mm}$ were contaminated with $5 \mathrm{~g}$ of oil ( $1 \mathrm{~g}$ or 38 drops per specimen) and left to age for 3 days before laundering. Each laundry load contained forty-five $500 \times 220 \mathrm{~mm}$ samples, including one experimental sample and 44 muslin buffers. The total mass of the samples in one load was $784 \mathrm{~g}$, and the total mass of oil on the experimental and oily buffer samples was $30 \mathrm{~g}$. The laundry process included a 10-min, small-load, heavy-duty cycle at $60^{\circ} \mathrm{C}$ with $195.5 \mathrm{~g}$ detergent added and two cold-water rinse cycles. Samples were dryer-dried for 15 min before cutting each sample into five specimens that were trimmed to size for FR testing $(80 \times 200 \mathrm{~mm})$. FR testing and statistical analyses were carried out as described here. Only damaged length was analyzed as a dependent variable.

\section{RESULTS AND DISCUSSION}

\subsection{Small-Scale Experiments}

Data representing all replications of the first experiment are summarized in Table 1, with each reported result representing the mean for 
TABLE 1. Summary of Flame Resistance (FR) Data in Small-Scale Laundry Experiment

\begin{tabular}{|c|c|c|c|c|c|}
\hline \multirow[b]{2}{*}{ Contamination } & \multirow[b]{2}{*}{ Detergent } & \multirow[b]{2}{*}{ Pre-Treatment } & \multicolumn{2}{|c|}{ Aramid } & \multirow{2}{*}{$\begin{array}{c}\text { FR Blend* } \\
\text { Damaged } \\
\text { Length Mean } \\
(\mathrm{mm})(S D)\end{array}$} \\
\hline & & & $\begin{array}{c}\text { Damaged } \\
\text { Length Mean } \\
(\mathrm{mm})(S D)\end{array}$ & $\begin{array}{c}\text { After-Flame } \\
\text { Mean (s) (SD) }\end{array}$ & \\
\hline \multirow[t]{8}{*}{ none } & industrial & none & $91.3(5.3)$ & 0 & $44.6(2.6)$ \\
\hline & & industrial & $92.9(7.4)$ & 0 & $47.1(3.5)$ \\
\hline & & domestic $\mathrm{A}$ & $86.7(10.3)$ & 0 & $46.8(2.5)$ \\
\hline & & domestic B & $91.1(5.9)$ & 0 & $47.1(3.0)$ \\
\hline & domestic liquid & none & $86.7(7.2)$ & 0 & 49.7 (4.9) \\
\hline & & industrial & $87.5(4.6)$ & 0 & $49.3(4.1)$ \\
\hline & & domestic $\mathrm{A}$ & $91.7(6.9)$ & 0 & $48.1(3.8)$ \\
\hline & & domestic B & $91.5(5.7)$ & 0 & $46.5(3.5)$ \\
\hline \multirow[t]{8}{*}{ oil } & industrial & none & $138.1(41.5)$ & $27.9(27.4)$ & $43.3(1.7)$ \\
\hline & & industrial & 104.5 (10.6) & $4.5(9.6)$ & $42.4(2.7)$ \\
\hline & & domestic A & $99.2(8.6)$ & 0 & $43.4(3.5)$ \\
\hline & & domestic B & $112.5(32.2)$ & $15.5(24.7)$ & $42.8(3.0)$ \\
\hline & domestic liquid & none & $110.1(26.8)$ & $4.1(15.1)$ & $41.6(2.2)$ \\
\hline & & industrial & $99.5(14.0)$ & 0 & $42.1(2.3)$ \\
\hline & & domestic $\mathrm{A}$ & $99.3(7.8)$ & 0 & $42.8(2.1)$ \\
\hline & & domestic B & $105.3(28.9)$ & $9.4(21.4)$ & $42.7(1.8)$ \\
\hline
\end{tabular}

Notes. *-after-flame times for all FR blend specimens were 0.

15 specimens. Analysis of variance indicated several interaction effects among fabric, contamination, detergent and pre-treatment, indicating that differences noted for one factor were in part determined by one or more of the other factors. Therefore, results are discussed here for each fabric separately. For the analyses on aramid fabrics, all main effects (contamination, detergent and pre-treatment were significant at $p<.01$; for the FR blend fabrics, the main effect for contamination was significant at $p<.01$ while the effects of detergent and pre-treatment were not significant.

\subsubsection{Aramid specimens}

When following the CGSB (Canadian General Standards Board) [5] flame resistance test procedure, damaged length of $100 \mathrm{~mm}$ or more is normally considered a failure. For aramid specimens, the damaged length of specimens contaminated with oil is consistently greater than that for uncontaminated specimens. For non-contaminated specimens, differences among detergents are small and there is little difference among pre-treatments, but it is interesting to note that some pre-treatments gave somewhat higher damaged lengths than without pre-treatment. In all cases for uncontaminated specimens, however, there was no after-flame.

For contaminated aramid specimens, the mean damaged length for many detergent/pre-treatment combinations represents failure. These same treatments all had long after-flames. All pre-treatments gave lower damaged lengths than did no pre-treatment, especially when the industrial detergent was used, and in general, specimens laundered with the liquid detergent had equal or lower damaged lengths than did those 
laundered with the industrial detergent. In each group, however, several individual specimens had damaged lengths over $100 \mathrm{~mm}$, and standard deviations for many groups of contaminated, laundered specimens are very high.

\subsubsection{FR viscose/aramid blend specimens}

The damaged lengths of contaminated FR blend specimens were consistently and significantly lower than the uncontaminated ones. There were no failing damaged lengths. As there was no after-flame on any specimen, this information is not included in Table 1. Such results might be considered surprising except that it confirms the results of the earlier field trials. This key finding, however, makes some results that follow more difficult to interpret.

In this case, analysis of variance indicated that neither detergent nor pre-treatment was a significant factor when considered alone, but there was a significant interaction effect among contamination, detergent and pre-treatment. Specimens laundered in the industrial detergent have slightly higher damaged lengths than those laundered with liquid detergent when oil is present and there is no pre-treatment. With pre-treatment, however, there is less difference between detergents. With no oil, specimens laundered in liquid detergent tend to have higher damaged lengths than when the industrial detergent was used.

Initial reaction to these results was to believe that oil was being removed more successfully from the FR blend than from aramid fabrics. Neither visual inspection of the laundered specimens nor observation of the flame resistance tests in progress confirmed that, however. Rather, an alternative explanation seemed possible: that energy from the burning oil on the specimens might be activating the FR finish on the blend fabrics, extinguishing the flame. To quickly test this hypothesis, five specimens of both the aramid and the FR blend were tested without contamination or laundering, and five of each were tested after contamination but without laundering. The results supported our alternative explanation. For aramid, as expected, the damaged lengths of the contaminated specimens (mean of 100.8 $\mathrm{mm}$ ) were higher than those of the uncontaminated (mean of 79.6). For the FR blend, however, the contaminated specimens had slightly lower damaged lengths (mean of 43.2) than the uncontaminated (mean of 45.6).

\subsection{Large-Scale Experiments}

Data representing results from two replications of the experiment in which larger samples were laundered in a domestic washing machine are summarized in Table 2, with each reported

TABLE 2. Summary of Flame Resistance (FR) Data for Large-Scale Laundry Experiment

\begin{tabular}{lcll}
\hline Fabric & $\begin{array}{c}\text { Contami- } \\
\text { nation }\end{array}$ & $\begin{array}{c}\text { Pre- } \\
\text { Treatment }\end{array}$ & $\begin{array}{c}\text { Damaged } \\
\text { Length } \\
\text { Mean } \\
(\mathbf{m m})(S D)\end{array}$ \\
\hline Aramid & none & none & ${ }^{\mathrm{a}} \mathbf{8 7 . 0}(5.6)$ \\
& & industrial & ${ }^{\mathrm{b}} \mathbf{7 9 . 8}(7.2)$ \\
& oil & nomestic & ${ }^{\mathrm{b}} \mathbf{8 0 . 4}(4.6)$ \\
& & industrial & ${ }^{\mathrm{b}} \mathbf{9} \mathbf{9 0 . 7}(6.7)$ \\
FR blend & none & none & ${ }^{\mathrm{a}} \mathbf{4 2 . 9}(7.1)$ \\
& & industrial & ${ }^{\mathrm{b}} \mathbf{5 2 . 5}(5.8)$ \\
& & domestic & ${ }^{\mathrm{b}} \mathbf{5 3 . 3}(6.6)$ \\
& oil & none & ${ }^{\mathrm{a}} \mathbf{4 2 . 4}(2.6)$ \\
& & industrial & ${ }^{\mathrm{b}} \mathbf{4 8 . 1}(6.6)$ \\
& & domestic & ${ }^{\mathrm{b}} \mathbf{4 8 . 6}(7.1)$ \\
\hline
\end{tabular}

Notes. a, b, c-for each fabric/contamination combination, means with the same superscript letter do not differ significantly from each other as determined by Duncan's post-hoc test.

result representing the mean for 10 specimens. As with the small-scale experiment, in three-way analysis of variance (ANOVA) there 
were significant differences between the two fabrics $(p<.001)$ and between contaminated and non-contaminated specimens $(p<.05)$, with interaction effects $(p<.001)$ between the two factors. In all cases, contaminated or not, aramid specimens had higher damaged lengths than did the FR blend specimens. The effect of contamination, however, depended on the fabric. Any pre-treatment effect was not significant alone, but there were interaction effects $(p<.001)$ between fabric and pre-treatment. As earlier, because of the interaction effects, one-way ANOVA results will be discussed separately for each fabric.

\subsubsection{Aramid specimens}

As in the small-scale experiments, contaminated (oily) aramid specimens had higher damaged lengths than the uncontaminated. For the contaminated specimens, both pre-treatments gave significantly lower damaged lengths than without pre-treatment. Unlike the small-scale experiments, however, none of the contaminated samples failed as a group, although some individual specimens that were contaminated and laundered without pre-treatment did fail. It seems that the full-scale laundry process allows for better removal of the oily dirt from the specimens than was the case in the Launder-Ometer, where it was possible that oil was being redeposited onto the specimens. Redeposition of oily dirt is a common laundry problem, however, and possibly accounts for failures in the worn garments contributed by workers early in the study and perhaps some of the field trial failures as well. This points to the necessity of carefully determining optimum load size and water/detergent/fabric ratio.

\subsubsection{FR Blend specimens}

When no pre-treatment was involved, contaminated and uncontaminated FR blend specimens had very similar damaged lengths. On the other hand for pre-treated specimens, the contaminated ones had lower damaged lengths than did the uncontaminated. Even more difficult to explain is the finding here that both pre-treatments significantly increase the damaged lengths of both contaminated and uncontaminated specimens. This may suggest that pre-treatment decreases flame resistance, or it may result from removing oily dirt that apparently triggers the FR mechanism. Careful interpretation of these results requires further investigation of several factors, including the location of any oil and/or pre-treatment residues within the fibres or on the fibre surfaces, as well as combustion mechanisms for oily and/or pre-treated fabrics.

\section{CONCLUSIONS AND RECOMMENDATIONS}

The experimental results reported here lend support to many of the conclusions and recommendations of earlier phases. More research is necessary in which several contributing factors are studied more closely before some of the results can be interpreted with confidence and definite conclusions drawn. Nevertheless, the following implications are suggested:

- The apparent ease of maintaining flame resistant properties of aramid/FR viscose blends was confirmed in this phase of the research. Rather than resulting from better removal of oily dirt, however, the validity of an alternative explanation was demonstrated, namely, that burning oil on the fabric may release energy and activate the FR mechanism of the FR finish. 
- For the aramid fabrics in this study, use of the liquid detergent and/or laundry pre-treatments improved oil removal from contaminated specimens more than use of the industrial detergent alone. Thus, use of liquid detergent and pre-treatment are recommended procedures. The pretreatments also decreased the flame resistance of the uncontaminated specimens in the small-scale experiments, however. Since this was not the case in the large-scale experiment, we conclude that redeposition of oily dirt onto aramids may be an issue if laundry conditions are not controlled carefully. Thus, for several reasons, we conclude that dry-cleaning or commercial laundering should be recommended for very dirty aramid garments wherever possible. For on-site laundry, load size and water/ detergent/fabric ratio need to be carefully controlled.

- Despite the apparent ability of oily FR blend fabrics to maintain their flame resistance in this study, the liquid detergent or laundry pre-treatments are recommended over the industrial detergent for these fabrics because removal of oily dirt is desirable for many other reasons, including hygiene considerations. Careful control of the laundry process is necessary, however, to ensure that pre-treatment products are removed and do not actually contribute to increased flaming combustion.

- For maximum removal of oily dirt in on-site laundry, use of hot water, pre-treatment of dirty areas, and consistent and appropriate amounts of detergent and water levels are recommended. Because of the necessity to better control laundry variables, it is recommended that one person be placed in charge of laundering dirty garments, rather than leaving the task up to individual workers.
Further research is recommended:

- To replicate this study with a broader range of FR materials, especially FR treated rayon and cotton fabrics, and to carefully control water hardness at levels recommended for maintenance of the various FR materials.

- To study the phenomenon of oil redeposition in laundering FR fabrics, to measure the actual residue levels in contaminated/ laundered fabrics, and to determine how oily dirt is held on or in the fibres of various FR fabrics; and

- To determine the mechanisms of combustion of oily dirt and pre-treatment products in combination with FR fabrics.

\section{REFERENCES}

1. Stull J, Dodgen CR, Connor MB, McCarthy RT. Evaluating the effectiveness of different laundering approaches for decontaminating structural fire fighting protective clothing. In: Johnson JS, Mansdorf SZ, editors. Performance of Protective Clothing, Fifth Volume (ASTM STP 1237). West Conshohocken, PA, USA: American Society for Testing and Materials (ASTM); 1996, p. 447-68.

2. Mäkinen $H$. The effect of wear and laundering on flame-retardant fabrics. In: McBriarty JP, Henry NW, editors. Performance of Protective Clothing, Fourth Volume (ASTM STP 1133). Philadelphia, PA, USA: American Society for Testing and Materials (ASTM); 1992. p. 754-65.

3. Chandler KMM, Crown EM. How clean is clean? Manager and worker perspectives on maintaining thermal protective workwear. In: Proceedings, International Textile and Apparel Association 2002 Annual Meeting [research abstract]. 
Retrieved June 7, 2004, from: www.itaaonline.org/ITAAnew/Proceedings 2002/Research Abstracts/104.htm

4. Crown EM, Bitner E, Feng A. How clean is clean? Controlled wear/decontamination study of thermal protective workwear. Proceedings, International Textile and Apparel Association 2002 Annual Meeting [research abstract]. Retrieved June 7, 2004, from: www.itaaonline.org/ITAAnew/ Proceedings2002/Research Abstracts/103.htm

5. Committee on Textile Test Methods and Terminology. Flame resistance-vertically oriented textile fabric or fabric assembly test (National Standard of Canada No. CAN-CGSB-4.2, No. 27.10-2000). Ottawa, Ont., Canada: Canadian General Standards Board; 2000.

6. International Organization for Standardization (ISO). Textile fabrics-burning behaviour - determination of ease of ignition of vertically oriented specimens (Standard No. ISO 6940 [E]:1984). Geneva, Switzerland: ISO; 1984. 